\title{
Software Development Multi-Sourcing Relationship Management Model (Sdmrmm) Protocol (A Systematic Literature Review)
}

\author{
Muhammad Salam ${ }^{1}$, Dr.Siffat Ullah Khan ${ }^{2}$ \\ ${ }^{1}$ (Department of Computer Science and IT, University of Malakand, Pakistan) \\ ${ }_{2}^{2}$ (Department, Computer Science and IT/Software Engineering, University of Malakand, Pakistan)
}

\begin{abstract}
CONTEXT: Software development multi-sourcing is a modern software engineering paradigm in which the software project is contracted out to multi-vendors. In software multi-sourcing, there is one client and many vendors involved in the outsourcing contract. The client relies on more than one outsourcing vendors for the fulfillment of the software development activity.

OBJECTIVE: The objective of this study is to develop SDMRMM to assist multi-sourcing vendor organizations in establishing, strengthening and maintenance of the relationships with fellow vendors and client in multisourcing situation. The model is for vendor to vendor and vendor to customer relationships in multi-sourcing paradigm.

METHOD: we have developed a systematic literature review (SLR) protocol for software development multisourcing relationships management model (SDMRMM) from vendor's perspective. SLR is based on a structured protocol and is therefore different from ordinary literature view.

EXPECTED OUTCOME: The anticipated outcome of this review will be a list of challenges faced by vendors in establishment and maintenance of relationships with Clint as well as fellow vendors in multi-vendors software outsourcing also critical success factors for establishment and maintenance of log-lasting relationships will be identified.
\end{abstract}

Keywords: Software Multisourcing; Multi-vendors outsourcing; Multisourcing relationships;

\section{Introduction}

Keeping in view global software development, software outsourcing is a new software engineering pattern[2].This research proposal describes the systematic literature review (SLR) [3] protocol which will be used to investigate that what are the challenges faced by vendors in the establishment and maintenance of multivendor outsourcing relationships and what are the critical success factors to be developed by vendors for the success of multi-source project. Identifying the challenges and critical success factors will help the vendors to establish and maintain long-lasting relationships with fellow vendors as well as clients. This may also help to ensure the successful outcomes of the multi sourced software projects.

Multi-sourcing is sometime called "multi-vendor outsourcing" OR "multi-supplier sourcing" [4].In multi-sourcing, the client has one outsourcing contract with multiple suppliers. A multi-sourcing paradigm allows a client to engage multiple vendors for various components of software project that are then jointly performed by multiple suppliers. since the work is performed together by multi vendors, this implies that a cooperative and competitive environment exists between the suppliers working jointly as shown in the Fig.1[4].

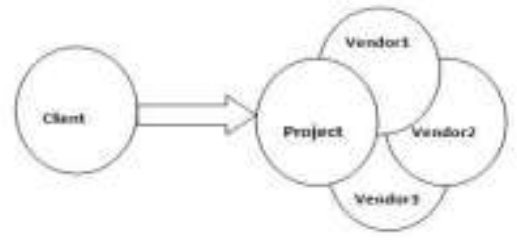

Fig.1 A typical multisourcing situation

There are five research questions as mentioned in section 3 of this protocol that have motivated the work reported in this research study.

The core purpose of this study is to build Software Development Multi-sourcing Relationship Management Model (SDMRMM).The model will help software outsourcing organizations (vendors) to establish sound and long lasting relationships with fellow vendors and clients in a software multisourcing.

The outsourcing may be unisourcing or multi-sourcing, prior to undertake software project outsourcing, the customer decides either to go for unisourcing or multi-sourcing. There are clear differences between the two approaches. The most important difference between unisourcing and multi-sourcing is that they require different 
internal management structures. Multisourcing will need wider internal management which has the capability to accommodate several suppliers at one time. Moreover there are other compulsions. In Unisourcing one party perform its promise whereby, in multi-sourcing more than one party held responsible in case of project failure. The cost and return factors along with risk of these two approaches are taken into consideration [5].Multi sourcing is preferred comparatively because it has many advantages for customers, for example, it creates a competing environment and decreases to maximum extent the risk of project failure, and enhance the quality of the software. Furthermore, multi sourcing is practiced when the suppliers feel that they are deficient in terms of capabilities[6]."Risk sharing is, hence, a common practice for parties adopting multiple outsourcing relationships"[7].Different studies show that trust is critical for all business relationships [8].However the focus of our study is on multi-vendors outsourcing relationships. A number of researchers have tried to address some of the issues of Multi-outsourcing, e.g. [9-11].Placement of an order in an environment, where many suppliers are working, needs proper attention and care, For reducing the average inventory cost, questions like when to place an order, how to order and how many order from each suppliers, are to be answered[6].The manager should establish and maintain productive and good working relationship among the members of virtual teams to achieve the predetermined results and goals, other studies in the literature have also showed the importance of trust in virtual teams [12].Establishing and maintaining good relationship with the outsourcing partners and suppliers helps the managers to share and transfer skills, and also ensure the trust worthiness and confidence of the suppliers on partners [13]."Recently, as global business environments have become increasingly complex and turbulent with the rise of off- shoring, firms started selecting and combining relationships with multiple service providers based in different countries as part of their sourcing strategy" [14]."New approaches and skills are required to effectively manage the increased volume and complexity of relationships between the various parties involved in multi-sourcing deals"[15].However, nobody has used the Systematic Literature Review (SLR) [3] approach in order to identify the challenges faced by vendors in multi-vendor outsourcing relationship and critical success factors to be developed by vendors for successful multi-vendor outsourcing relationships. "A systematic literature review is a means of identifying, evaluating and interpreting all available research relevant to a particular research question, or topic area, or phenomenon of interest"[16]."systemic literature review (SLR) has become an important research methodology in software engineering, since the introduction of evidence-based software engineering(EBSE) in 2004"[17].The purpose of this study is to develop Software Development Multi-sourcing Relationships Management Model (SDMRMM) the model is useful for software vendors which works in multi-vendors outsourcing. During the model and protocol development I have studied a number of systemic literature reviews $[18,19]$.

\section{Background of multi-sourcing}

Multi sourcing is the combination of two words-'Multi' and 'Sourcing'. Hence Multi mean many and "Sourcing refers to the act of transferring work, responsibilities and decision rights to someone else" or in other words we can say that "Sourcing is the act of transferring work from one entity to another" so we can say that multi sourcing is the transferring of work (software) from one entity (Client) to multiple entities (Vendors).According to [20] there are three components of outsourcing 1) Client 2) Vendor 3) Project, The organization transferring the work is referred to as the client, the organization that conducts the work and makes decisions is the vendor, and the scope of the work is captured in a project as shown in Fig.2

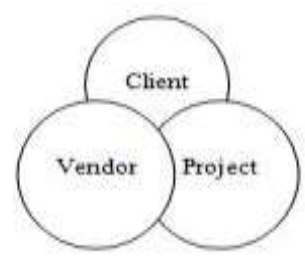

Fig.2

"Software development outsourcing is defined as a situation where a company (a client) contracts out all or part of its software development activates to another company (a vendor), who provides agreed services for remuneration" [21]. "In today's global services outsourcing arena, increasing numbers of companies adopt multi-sourcing, that is, they select and combine information technology (IT) and business services from multiple providers" [14]. "Multi-sourcing is when you outsource functions to more than one vendor. Multi-sourcing involves breaking up the project into several components that can be handled by independent vendors and usually occurs during a total outsourcing project. Multi-sourcing encourages competition between vendors and creates a shared objective to Perform at high levels" [20]. Components of multi-sourcing Relationship are shown in the following Fig.1. 
According to [22] are various types of outsourcing which can be divided into two main categories as shown in the Fig.3

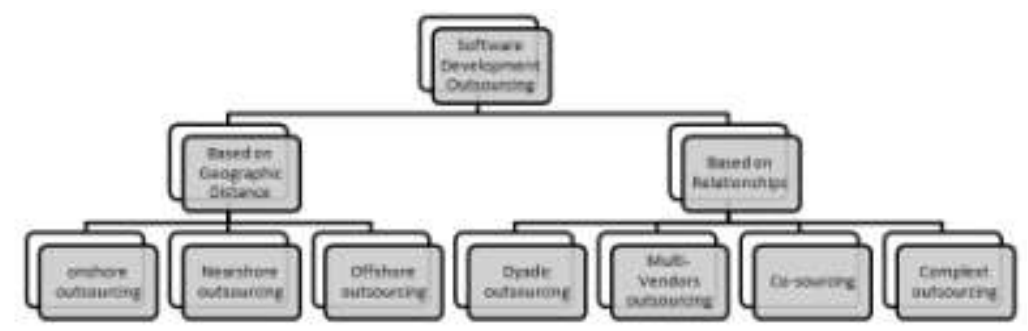

Fig.3 various types of outsourcing

The research work reported in this study is related to multisourcing relationships management from vendor to vendor (V2V) and from vendor to customer (V2C). The research is based on systematic literature review. The protocol for SDMRMM is developed on the basis of five research questions which are mentioned in section 3 of this protocol.

\section{Research Questions}

There are five research questions that have motivated the work reported in this research proposal. RQ1. What are the challenges/barriers, as identified in the literature, faced by vendor's in the establishment and maintenance of relationships with client as well as the fellow vendors in multi-vendors software outsourcing situation?

RQ2. What are the critical success factors, as identified in the literature, to be developed by vendors for the establishment and maintenance of long-lasting relationships with client as well as the fellow vendors in multivendors software outsourcing situation?

RQ3. What are the challenges/barriers, as identified in the outsourcing industry, faced by vendors in the establishment and maintenance of relationships with client as well as the fellow vendors in multi-vendors software outsourcing situation?

RQ4. What are the critical success factors, as identified in the outsourcing industry, to be developed by vendors for the establishment and maintenance of long-lasting relationships with client as well as the fellow vendors in multi-vendors software outsourcing situation?

RQ5. What are the real-world practices for addressing the identified success factors and barriers in multivendors software outsourcing situation?

For RQ1 and RQ2 the identification of CBs (Critical Barriers) and CSFs (Critical Success Factors), we will use systematic literature review (SLR). We will conduct empirical study (questionnaire survey) for the validation of SRL findings and to answer research questions RQ3, RQ4 and RQ5. The Software Development multi-sourcing relationship Management Model (SDMRMM) will be developed using SLR guidelines[3, 16] this model will help software outsourcing organizations (vendors) to indentify the challenges/barriers in establishment and maintenance of relationships with client as well as fellow vendors in multi-vendors software outsourcing, the proposed model will be useful for establishment and maintenance of log-lasting relationships with client and fellow vendors.

\section{Systematic Literature Review Protocol for SDMRMM}

A systematic literature review is a new research methodology, it is a way of identifying, evaluating and interpreting all available research relevant to a particular research question, or topic area, or phenomenon of interest [16]. Now a a day's systematic literature review has become an important research methodology [17]. SLR has the following main three phases.

- Planning the review

- Conducting the review

- Reporting the review

The expected outcomes of this review will be the identification of different CSFs and CBs that play important role in software development multisourcing relationships.

\subsection{Constructing Search Term}

The following details will help in designing a search term relevant to our research questions.

- Population: Software Development multisourcing relationship, multi-vendors software outsourcing relationships, multi supplier software outsourcing, client and vendors.

- Intervention: challenges, barriers, critical success factors, establishment of relationships, maintenance relationships. 
- Outcomes of relevance: establishment and maintenance of long-lasting relationships, identification of challenges/barriers in establishment and maintenance of relationships with client as well as fellow vendors in multi-vendors software outsourcing, Software Development Multi-sourcing Relationship Management Model (SDMRMM), long-lasting relationships with clients, long-lasting relationships with fellow vendors.

- Experimental Design: Empirical studies, systematic literature review, theoretical studies, case studies, expert's opinions, etc.

Examples of the Research Questions containing the above details are:

RQ1:

- [Challenges/barriers]

- [Software Multi-sourcing Relationships] INTERVENTION

- Faced by vendor

- $\quad$ in the establishment and maintenance of relationships with client as well as the fellow vendors in multi-vendors software outsourcing situation] $\gg$ OUTCOMES OF RELEVANCE

\subsection{Trail search}

\section{V. search strategy}

A trial search was conducted using the following search string on selected digital libraries as shown section $\mathbf{5 . 4}$ of this protocol.

(Multisourcing OR "software multisourcing” OR "multi vendors outsourcing" OR "multi Supplier outsourcing" OR "dual sourcing”) AND ("multi-vendors relationships" OR "multi-vendors outsourcing" OR "outsourcing relationships").

\begin{tabular}{|l|l|l|}
\hline S.No & Digital library & Search Conduction Date \\
\hline IEEE Explore & 15,55 & 26 April 2012 \\
\hline ACM Digital Library & 1214 & 26 April 2012 \\
\hline Google Scholar & 232 & 26 April 2012 \\
\hline Science Direct & 1084 & 26 April 2012 \\
\hline Site Seer Digital Library & 1116 & 26 April 2012 \\
\hline Springer link & $236+247$ & 26 April 2012 \\
\hline
\end{tabular}

Table 2: Trail Search Results

The papers retrieved through this search string will be used as a guide for the development and validation of the major search terms.

\subsection{Indentifying search term}

The following search strategy is used for the construction of search terms.

a) Use the Research Questions for the derivation of major terms, by identifying population, intervention and outcome;

b) For these major terms, find the alternative spellings and synonyms;

c) Verify the key words in any relevant paper;

d) Use of Boolean Operators OR, AND

\section{Results for (a)}

RQ1: Multisourcing, software development multisourcing relationships, challenges, barriers, client, vendors, Establishment and maintenance of multisourcing relationships.

RQ2: Multisourcing, Software development multisourcing relationships, success factors, client, vendors, Establishment and maintenance of Multisourcing relationships

\section{Results for (b)}

RQ1:

Multisourcing :( Multisourcing OR "Multi-vendor outsourcing” OR “multiple-supplier sourcing” OR "dual sourcing" OR "multiple sourcing")

Software development multisourcing relationships: ("multi-vendor relationships" OR "multisourcing relationships" OR "multiple supplier relationships" OR "dual sourcing relationships" OR "software multisourcing relationships" OR "software multi-vendor relationships" OR "multi-vendor collaboration" OR "multi vendor coordination" OR "multi-vendors alliance" OR "multi-vendors partnerships" OR "multisourcing contract")

Challenges: (challenges OR barriers OR problems OR complications)

Barriers: (risks OR failures OR barriers "critical factors" OR "risk analysis" OR “critical factors") 
Client: (customer OR clients OR users)

Vendors: ("service providers" OR supplier OR developer)

Establishment and maintenance of multisourcing relationships: ("Building multi-sourcing relationships" OR "establishing multi-vendors relationships" OR "multi-sourcing relationships management" OR "strengthening multi vendors relationships")

RQ2: "dual sourcing")

Multisourcing :(Multisourcing OR "Multi-vendor outsourcing” OR “multiple-supplier sourcing” OR

Software development multisourcing relationships: ("multi-vendor relationships" OR "multisourcing relationships" OR "multiple supplier relationships" OR "dual sourcing relationships" OR "software multisourcing relationships" OR "software multi-vendor relationships" OR "multi-vendor collaboration" OR "multi vendor coordination" OR "multi-vendors alliance" OR "multi-vendors partnerships" OR "multisourcing contract")

Success factors :( "Success elements" OR factors OR "success factors" OR "success parameters" OR "key factores" OR "important factors")

Client: (customer OR clients OR users)

Vendors: ("service providers" OR supplier OR developer)

Establishment and maintenance of multi-sourcing relationships: ("Building multi-sourcing relationships" OR "establishing multi-vendors relationships" OR "multi-sourcing relationships management" OR "strengthening multi vendors relationships")

\section{Results for $(\mathbf{C})$}

Multi-sourcing, software multi-sourcing, multi vendors outsourcing, multi Supplier outsourcing, dual sourcing, multi-vendors relationships, multi-vendors outsourcing, vendors relationships, critical success factors, factors, challenges, software multi-sourcing models, dual sourcing relationships, vendors to vendors relationships, vendors to client relationships, supplier relationship management.

\section{Results for (d) \\ RQ1.}

("Multi-vendor outsourcing" OR "multiple-supplier sourcing" OR "dual sourcing" OR "multiple sourcing")AND(("multi-vendor relationships" OR "multisourcing relationships" OR "multiple supplier relationships" OR "dual sourcing relationships" OR "software multi-sourcing relationships" OR "software multi-vendor relationships" OR "multi-vendor collaboration" OR "multi vendor coordination" OR "multivendors alliance" OR "multi-vendors partnerships" OR "multisourcing contract")OR(challenges OR "barriers" OR "problems" OR "complications" ) OR("risks" OR failures OR barriers "critical factors" OR "risk analysis" OR "critical factors" ) OR ("customer" OR "clients" OR "users") OR("service providers" OR "supplier" OR "developer")OR("Building multi-sourcing relationships" OR "establishing multi-vendors relationships" OR "multi-sourcing relationships management" OR "strengthening multi vendors relationships"))

RQ2.

("Multi-vendor outsourcing" OR "multiple-supplier sourcing" OR "dual sourcing" OR "multiple sourcing")AND ((multi-vendor relationships" OR "multisourcing relationships" OR "multiple supplier relationships" OR "dual sourcing relationships" OR "software multi-sourcing relationships" OR "software multi-vendor relationships" OR "multi-vendor collaboration" OR "multi vendor coordination" OR "multivendors alliance" OR "multi-vendors partnerships" OR "multisourcing contract") OR Success factors :( "Success elements" OR factors OR "success factors" OR "success parameters" OR "key factors" OR "important factors") OR (Customer OR clients OR users) OR ("Service providers" OR "supplier" OR "developer") OR ("Building multi-sourcing relationships" OR "establishing multi-vendors relationships" OR "multi-sourcing relationships management" OR "strengthening multi vendors relationships"))

\subsection{Search term break up}

We will first use the search strings RQ1 and RQ2 mentioned in 3.2(d) as our search terms. As Some of the databases don't allow the lengthy search strings we will split the search term into Smaller sub strings and will do separate search for each of these search strings. Finally we will summarize the search results by removing the redundancy. The breakup of the RQ1 and RQ2 are mentioned in the form smaller strings mentioned below.

Search string 1

("Multi-vendor outsourcing" OR "multiple-supplier sourcing" OR "dual sourcing" OR "multiple sourcing")AND("multi-vendor relationships" OR "multisourcing relationships" OR "multiple supplier 
relationships" OR "dual sourcing relationships" OR "software multi-sourcing relationships" OR "software multi-vendor relationships" OR "multi-vendor collaboration" OR "multi vendor coordination" OR "multivendors alliance" OR "multi-vendors partnerships" OR "multisourcing contract")

Search string 2

("Multi-vendor outsourcing" OR "multiple-supplier sourcing" OR "dual sourcing” OR "multiple sourcing")AND (Challenges OR barriers OR problems OR complications)

Search string 3

("Multi-vendor outsourcing" OR "multiple-supplier sourcing" OR "dual sourcing" OR "multiple sourcing")AND (Risks OR failures OR barriers "critical factors" OR "risk analysis" OR "critical factors")

Search string 4

("Multi-vendor outsourcing" OR "multiple-supplier sourcing" OR "dual sourcing" OR "multiple sourcing")AND (Customer OR clients OR users)

\section{Search String 5}

("Multi-vendor outsourcing" OR "multiple-supplier sourcing" OR "dual sourcing" OR "multiple sourcing")AND (Service providers OR supplier OR developer)

Search string 6

("Multi-vendor outsourcing" OR "multiple-supplier sourcing" OR "dual sourcing" OR "multiple sourcing")AND ("Building multi-sourcing relationships" OR "establishing multi-vendors relationships" OR "multi-sourcing relationships management" OR "strengthening multi vendors relationships")

Search string 7

("Multi-vendor outsourcing" OR "multiple-supplier sourcing" OR "dual sourcing" OR "multiple sourcing")AND Success factors :( "Success elements" OR factors OR "success factors" OR "success parameters" OR "key factors" OR "important factors")

\subsection{Resources to be searched}

The following digital libraries and databases will be searched.

- IEEE Explore

- ACM Digital Library

- Google Scholar

- Science Direct

- Site Seer Digital Library

- Springer link

\subsection{Search Constraints and validation}

The above selected digital libraries will be searched against each search string (string to string 7) with out applying any Date boundaries.

\subsection{Search Documentation}

The search results will be documented in a table having the following information.

- Search conduction date

- Name of digital library

- Searched string used

- Date Constrain

- Total publication found

- Initial selection

- Final selection

\subsection{Search Result Management}

The records all the candidate papers from the digital libraries are kept in a directory. In which the Record of each digital library is stored in separate directory, where each page is saved as .html page.

To be on safe side the the backup of the search results are also stored on Google Drive. 


\section{VI. publication selection}

\subsection{Inclusion Criteria}

Inclusion criteria are used to select only that literature (research papers / reports / books) found in the search results obtained. We will only select those research papers related to augmented reality. The inclusion criteria are listed as below:

- Research studies and articles that are related to our protocol

- Research work that describe software multi-sourcing

- Research work that describes difficulties difficulties in software multi-sourcing

- Research work that identifies software multi-sourcing relationships

- Research papers and studies that describe multi-sourcing relationships

- Paper that describes the challenges of multi-sourcing relationships

- Research work that describes multi vendor's relationships.

- Research papers that describes multi supplies relationships management.

- Research papers that describes barriers and challenges in multi-sourcing relationships with clients and fellow vendors.

- Research papers and articles that describes critical success factors for the establishment and maintenance of long-lasting relationships with clients and fellow vendors in multi vendor software outsourcing.

\subsection{Exclusion Criteria}

Exclusion criteria are used to decide which piece of literature (research papers / reports / books) found by the search term will not be selected for review. The criteria are listed below:

- Research work that is not relevant to the research questions will be excluded.

- Literature that don't describe multi-sourcing relationships.

- Research work other then multi-sourcing and multi vendors.

\subsection{Selecting primery sourecs}

Primary sources will be initially selected by analyzing the title, keywords and abstracts of searched literature. This review will exclude / ignore those searched literature which have no relevance to the research questions. The primary sources chosen during this initial selection process will be checked against the above inclusion / exclusion criteria by reviewing through full text of the research papers. If any uncertainty occurs regarding the inclusion / exclusion decision, the case will be sent to the secondary reviewer. The process will be checked by the third reviewer. Inclusion / exclusion decision record regarding each primary source will be maintained properly. This will include the justification whether or not the primary source has been included in the final review.

\section{VII. publication quality assessment}

The conduction of quality measurement will be performed after the final selection of publications. The quality checklist contains the following questions:

- Is it clear how the challenges/barriers faced by vendors in the establishment and maintenance of relationships with clients as well as the fellow vendors in multi-vendors software outsourcing situation were identified?

- Is it clear how the critical success factors to be developed by the vendors for establishment and maintenance of long-lasting relationships with client as well as the fellow vendors in multi-vendors software outsourcing situation were identified?

- Each of the above questions will be marked as 'YES', 'NO', 'Partially' or N.A'.

\subsection{Primery study data}

\section{Data extraction strategy}

The core aim of SDMRMM protocol is to extract the data which satisfies the RQ1 and RQ2.

The following data will be extracted from each selected research publication.

Date of review, title, authors, references, database , methodology, sample population, publication quality description, organization type, company size, location of analysis, year, CBs (Critical Barriers), CSFS (Critical success factors).

\subsection{Data Extraction process}

The review will be undertaken by a single researcher, who will be responsible for the data extraction. A secondary reviewer and tertiary reviewer will be approached for guidance in case of an issue regarding the data extraction. The reliability test will be performed after the data extraction process by the primary reviewer. The secondary reviewer will select few publications randomly from the list of publication already chosen by the primary reviewer. The secondary reviewer will independently extract the data from the randomly selected 
publication. The results will then be compared with the results produced by the primary reviewer. The tertiary reviewer will be approached for guidance during conducting the review.
Primary Reviewer:
Mr.Muhammad Salam
Dr. Siffat Ullah Khan

Secondary Reviewer:

\subsection{Data storage}

The summarized data for each publication will be kept as a Microsoft Word/pdf document and will be stored as soft form in personal laptops by all authors. A backup are kept at Google Drive.

\section{Data synthesis}

The synthesis of data will also be categorized into two parts as we have two research questions. For the RQ1, the data will be synthesized by creating one summary table having the columns(S.No, challenges/barriers, Frequency, Percentages) showing the list of all the factors along with their frequencies and percentages. For the RQ2, the data will be synthesized by creating one summary table having the columns (S.No, CSF, Frequency, Percentages) showing the list of all the factors along with their frequencies and percentages.

\section{Divergences}

In case of any divergence from the protocol that may occur during the study will be recorded in an Appendix to this report.

\section{Acknowledgment}

We are thankful Software Engineering Research Group at University of Malakand (SERG_UOM) for the Review and their valuable comments in validation process of the protocol.

\section{References}

[1] S. U. Khan, M. Niazi, and N. Ikram, "Software Development Outsourcing Relationships Trust: A Systematic Literature Review Protocol," presented at Evaluation and Assessment in Software Engineering, EASE 2010, Keele University, UK, 2010.

[2] S. U. Khan, M. Niazi, and R. Ahmad, "Barriers in the Selection of Offshore Software Development Outsourcing Vendors: An Exploratory Study using a Systematic Literature Review," Information and Software Technology,(Elsevier), doi: 10.1016/j.infsof.2010.08.003, 2010.

[3] B. Kitchenham and C. Charters, "Guidelines for performing Systematic Literature Reviews in Software Engineering, Keele University and Durham University Joint Report," EBSE 2007-001, 2007.

[4] H. S. Kehal and V. P. Singh, Outsourcing and offshoring in the 21st Century: A Socio-Economic Perspective.

[5] J. K. HALVEY and B. M. MELBY, INFORMATION TECHNOLOGY OUTSOURCING TRANSACTIONS PROCESS, STRATEGIES, AND CONTRACTS, 2ND EDITION ed: John Wiley \& Sons, Inc., Hoboken, New Jersey.Published simultaneously in Canada.

[6] G. Wang, Z. Jiang, Z. Li, and W. Liu, " Supplier selection and order splitting in multiple-sourcing inventory systems," Frontiers of Mechanical Engineering in China, vol. 3, pp. 23-27.

[7] R. Sharma, S. Apoorva, V. Madireddy, and V. Jain, "Best Practices for Communication between Client and Vendor in IT Outsourcing Projects," Journal of Information, Information Technology, and Organizations, vol. 3, 2008.

[8] P. Kanawattanachai and Y. Yoo, "Dynamic Nature of Trust in Virtual Teams," The Journal of Strategic Information Systems, vol. 11, pp. $187-213,2002$

[9] O. d. Gaudemar, "Multi-sourcing: benefits and challenges," vol. 2011

[10] "Egham, UK, August 27, 2009 - Gartner, Inc Three Pitfalls of Multisourcing," vol. 2011,27 August 2009.

[11] v. b. kartha, "Multi-Sourcing from Best of Breed Providers:Perspectives for BPO," vol. 2011, January 2007.

[12] P. Kanawattanachai and Y. Yoo, "Dynamic Nature of Trust in Virtual Teams," Sprouts: Working Papers on Information Systems, vol. 2, 2002.

[13] ShitaoYanga, JianYangb, and L. Abdel-Malek, "Sourcing with random yields and stochastic demand: A newsvendor approach," Computers \& Operations Research, vol. 34, pp. 3682 - 3690, 2007.

[14] N. Levina and N. Su, "Global Multisourcing Strategy: TheEmergence of a Supplier Portfolio in Services Offshoring," Decision Sciences, vol. Volume 39 Number 3, pp. 541-570, August 2008.

[15] " Meeting the Challenges of Supplier Relations in a Multisourcing Environment."

[16] B. Kitchenham, "Procedures for Performing Systematic Reviews," Keele University Technical ReportTR/SE0401, 2004.

[17] H. Zhang, M. A. Babar, and P. Tell, "Identifying relevant studies in software engineering," Information and Software Technology, vol. 53, pp. 625-637, 2011.

[18] P. Brereton, B. A. Kitchenham, D. Budgen, M. Turner, and M. Khalil, "Lessons from applying the systematic literature review process within the software engineering domain," The Journal of Systems and Software, pp. 571-583, 2007.

[19] H. Zhang, M. A. Babar, and P. Tell, "Identifying relevant studies in software engineering," Information and Software Technology, vol. 53, pp. 625-637, 2011

[20] M. J. Power, K. C. Desouza, and C. Bonifazi, The Outsourcing Handbook How to implement a successful outsourcing process: First published in Great Britain and the United States in 2006 by Kogan Page Limited.

[21] M. Ali-Babar, J. Verner, and P. Nguyen, "Establishing and maintaining trust in software outsourcing relationships: An empirical investigation," The Journal of Systems and Software, vol. 80, pp. 1438-1449, 2007.

[22] S. U. Khan and M. Niazi, "Systematic Literature Review Protocol for Software Outsourcing Vendors Readiness Model (SOVRM), Technical Report: TR/08-01, ISSN: 1353-7776, School of Computing and Maths, Keele University, UK," 2008.

[23] M. J. Gallivan and W. Oh, "Analyzing IT Outsourcing Relationships as Alliances among Multiple Clients and Vendors," presented at Proceedings of the 32nd Hawaii international Conference on System Sciences, Hawaii, USA, 1999.

[24] V. Imsland, "The Role of Trust in Global Software Outsourcing Relationships," in Department of Informatics: UNIVERSITY OF OSLO, 30th July 2003 